\title{
Weaving through a cryptic species: Comparing the Neotropical ants Camponotus senex and Camponotus textor (Hymenoptera: Formicidae)
}

\author{
Eduardo Gonçalves Paterson Fox ${ }^{\mathrm{a}, *}$, Daniel Russ Solis ${ }^{\mathrm{d}}$, Cristiano Lazoski ${ }^{\mathrm{b}}$, William Mackay ${ }^{\mathrm{c}}$

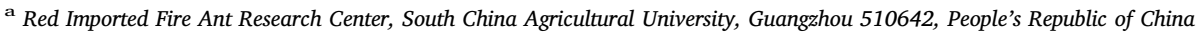 \\ b Departamento de Genética, Instituto de Biologia, Universidade Federal do Rio de Janeiro, RJ, Brazil \\ c Biodiversity Collections, Department of Biological Sciences, The University of Texas, El Paso, TX 79968, USA \\ d Centro de Estudos de Insetos Sociais, Sao Paulo State University, 24A 1515, Bela Vista 13506-900, Rio Claro, SP, Brazil
}

\section{A R T I C L E I N F O}

\section{Keywords:}

Ant systematics

Myrmecological diversity

Body hairs

Bristles

Chemotaxonomy

Molecular ecology

Cuticular waxes

Ultrastructure

\begin{abstract}
A B S T R A C T
Camponotus senex (Fr. Smith 1858) and Camponotus textor Forel, 1899 are commonly confused species in the New World tropics. We provide morphological characteristics based on the larvae and adults, behavioural differences, together with evidence from molecular markers (cuticular hydrocarbon profiles, venom differences, nuclear ribosomal ITS-1, and mtDNA COI sequence comparisons) to separate the two species, demonstrating they are not immediately closely related. In conclusion we suggest new reliable morphological characters which can benefit from deeper phenetic analysis, and support the contextual usefulness of non-morphological tools in resolving sibling ant species.
\end{abstract}

\section{Introduction}

The taxonomic study of ants (Hymenoptera: Formicidae) is greatly complicated by an abundance of cryptic species (Seifert 2009), i.e. evolutionarily independent populations which are or were once united as a single nominal species that are difficult to separate visually (Bickford et al., 2007). Mayr (1963) proposed that cryptic species should be more abundant in those taxa not relying on visual cues for conspecific recognition, thus being the case with ants as they generally rely on chemical communication and in fact abound in morphologically similar species.

Camponotus textor Forel, 1899 is a South American formicine weaver ant that has been historically a subspecies of the opportunistic cavity-dweller Camponotus senex (Fr. Smith, 1858) until being validated as a separate species based on behavioural, biological, and morphological grounds (Longino, 2006). A recent study by Ramalho et al. (2016a) quantified the divergence of a mitochondrial gene (COI) between these two species, while remarking of the extreme differences in their nests' construction and physical location, and proposed using COI as a molecular technique to distinguish between the two species. It is a remarkable fact that so similar sympatric species will in fact behave so differently, illustrating the issue of sibling species in ants. What other differences exist between the two species that could facilitate a diagnosis, and clarify their evolutionary history?

Several authors have shown that the larvae of hymenopterans can provide invaluable insights into the phylogenetic relationships (Finlayson,
1967; Wheeler and Wheeler, 1976; Schultz and Meier, 1995) and may provide useful morphological characters for taxonomy, particularly in ants (Wheeler and Wheeler, 1976; Pitts et al., 2005; Fox et al., 2012). Other promising techniques for species separation are chemical tools such as venom and cuticular hydrocarbon chemistry (Jones and Blum, 1982; Vander Meer and Lofgren, 1988; Seifert, 2009) and molecular markers such as some well-conserved mitochondrial and nuclear DNA regions including COI gene and ribosomal ITSs (Seifert, 2009). Taken together, these tools can help elucidate problems with the alpha-taxonomy of ants in which many species cannot be separated by means of classical analysis of adult morphology, and sometimes the morphological characters include traits that are difficult to evaluate or invalidated by intraspecific variation (e.g. Seifert, 2009).

The present investigation thus aimed to add resolution to the differences between $C$. senex and C. textor in terms of mature larval morphology, adult morphology, hydrocarbon chemistry, mtDNA, and nuclear DNA.

\section{Materials and methods}

\subsection{Sampling and morphological data}

Nests of Camponotus textor and C. senex were obtained on the campus of São Paulo State University in Rio Claro, São Paulo, Brazil.

\footnotetext{
* Corresponding author.

E-mail address: ofoxofox@gmail.com (E.G.P. Fox).
} 

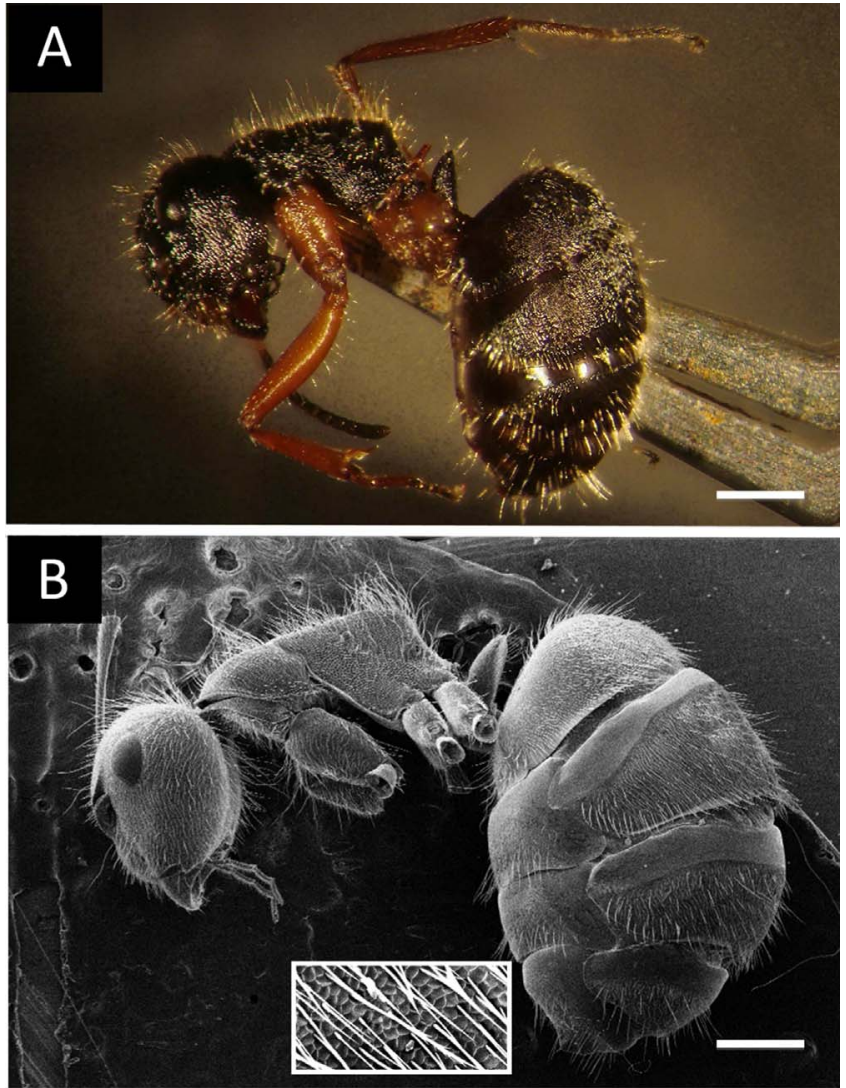

Fig. 1. Major worker of Camponotus textor in profile A) killed by freezing without fixation, and B) seen by scanning electron microscopy (SEM) on stub; inset is a $100 \times 200 \mu \mathrm{m}$ close-up on gastral surface. Size of scale bars $(\mu \mathrm{m}): 650 ; 700$.
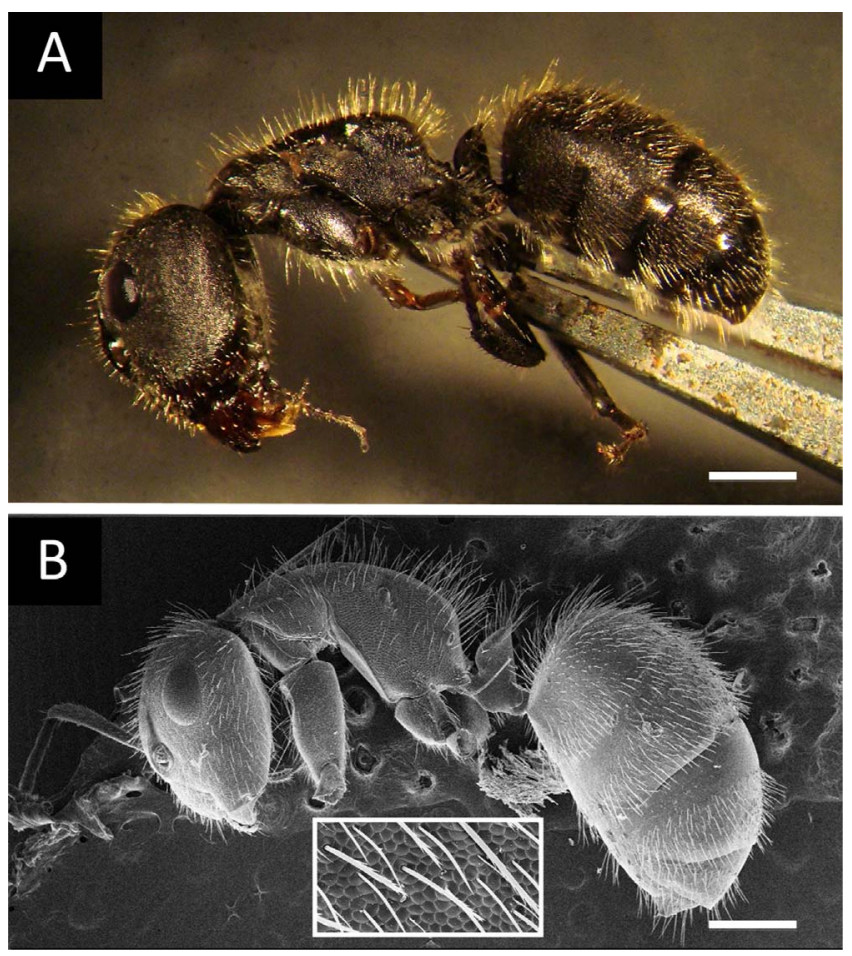

Fig. 2. Major worker of Camponotus senex in profile A) killed by freezing without fixation, and B) seen by scanning electron microscopy (SEM) on stub; inset is a $100 \times 200 \mu \mathrm{m}$ close-up on gastral surface. Size of scale bars $(\mu \mathrm{m})$ : $500 ; 600$.
Silken nests of $C$. textor were recovered from two different trees, while two nests of $C$. senex were sampled from inside active mounds of the termite Heterotermes tenuis (Hagen). The ant nests were not in their reproductive period, meaning that no reproductive larvae or adults were seen. We obtained abundant worker larvae and adults from the nests for comparative studies. Mature larvae will acquire a characteristic shape, particularly after weaving a cocoon, which are termed as prepupae - we have analysed only mature larvae for the present investigation, which were selected based on head capsule width and morphology (e.g. pseudopalps) of field-obtained prepupae. It should be kept in mind that $C$. textor is only weakly polymorphic and in fact no major workers were perceived (Longino, 2006), thus workers were compared irrespective of relative size. Camponotus senex on the other hand is dimorphic and only the largest workers were used. Larvae were collected irrespective of length size as we lack specific data on the relative larval sizes of workers, but from experience with other Camponotus we expect significant length differences to arise only at the prepupal stage (e.g. Solis et al., 2010). It should be emphasized that mature larvae of formicine ants are marked by the presence of pseudopalps.

These collections were supplemented by museum specimens for an ampler comparison. Species were identified, apart from its unique nest constructions, based on comparison with the original type material: (i) "Formica senex", 2 minor workers and 1 female (deposit B. M. Type 11.656 at the British Museum of Natural History, London); and (ii) Camponotus senex race textor, 1 major worker, 5 minor workers, and 3 females (deposited at the Muséum d'Histoire Naturelle, Genéve,). Some images of (i) can be found online at http://www.antwiki.org/wiki/ Camponotus_senex; images of (ii) can be found at http://www.antwiki. org/wiki/Camponotus_textor

Voucher specimens of both species are deposited in Brazil in the entomological collections of Museu Nacional do Rio de Janeiro and Instituto Biológico de São Paulo, and in the United States in the Biodiversity Collections of the University of Texas, El Paso, Texas. Ant specimens were mounted and photographed for analysis. Both adult major and minor workers from both species were prepared for scanning electron microscopy (SEM) analysis following the methods of Solis et al. (2010), except that $80 \%$ ethanol was used in fixation. Collected specimens of each species were compared to their counterpart museum deposited specimens for the minimum and maximum measurement intervals for: head length (taken from occipital margin to border clypeus); antennal scape length; eye length (taken from posterior to anterior borders) using a micrometric eyepiece mounted on binoculars.

Mature larvae of $C$. senex were measured, photographed, and analysed by light and SEM using also the methods of Solis et al. (2010), except that samples were fixed and preserved in ethanol $80 \%$. Results were compared with acquired data from $C$. textor from Solis et al. (2009). Morphological descriptions of larvae follow the terminology of Wheeler and Wheeler (1976) with modifications from Solis et al. (2010). Obtained image data available in Fox et al. (2017).

\subsection{Hydrocarbon analyses}

Live workers ( $\mathrm{N}=3$ from three different nests) were directly immersed in 100\% HPLC-grade hexane (MERCK) for extraction of cuticular hydrocarbons, followed with the concentration and analysis of the extract by gas chromatography and mass spectrometry (GC-MS) as previously described in Fox et al. (2012), but with a relevant correction shown in the following quotation: the extraction samples were analysed "by injecting $1 \mu \mathrm{L}$ of each extract in a GCMS-QP2010 (SHIMADZU) system equipped with a RTX-5MS silica capillary column $(30 \mathrm{~m} \times 0.25 \mathrm{~mm} \times 0.25 \mu \mathrm{m})$. Helium was the carrier gas, used at a flow rate of $1.0 \mathrm{~mL} / \mathrm{min}$ on split[less] mode. The MS were taken at $70 \mathrm{eV}$, and scanning speed was set to 1228 , from $\mathrm{m} / \mathrm{z} 50$ to700. The interface temperature was maintained at $280{ }^{\circ} \mathrm{C}$. The injector temperature was $250^{\circ} \mathrm{C}$. Oven temperature was programmed to increase at 

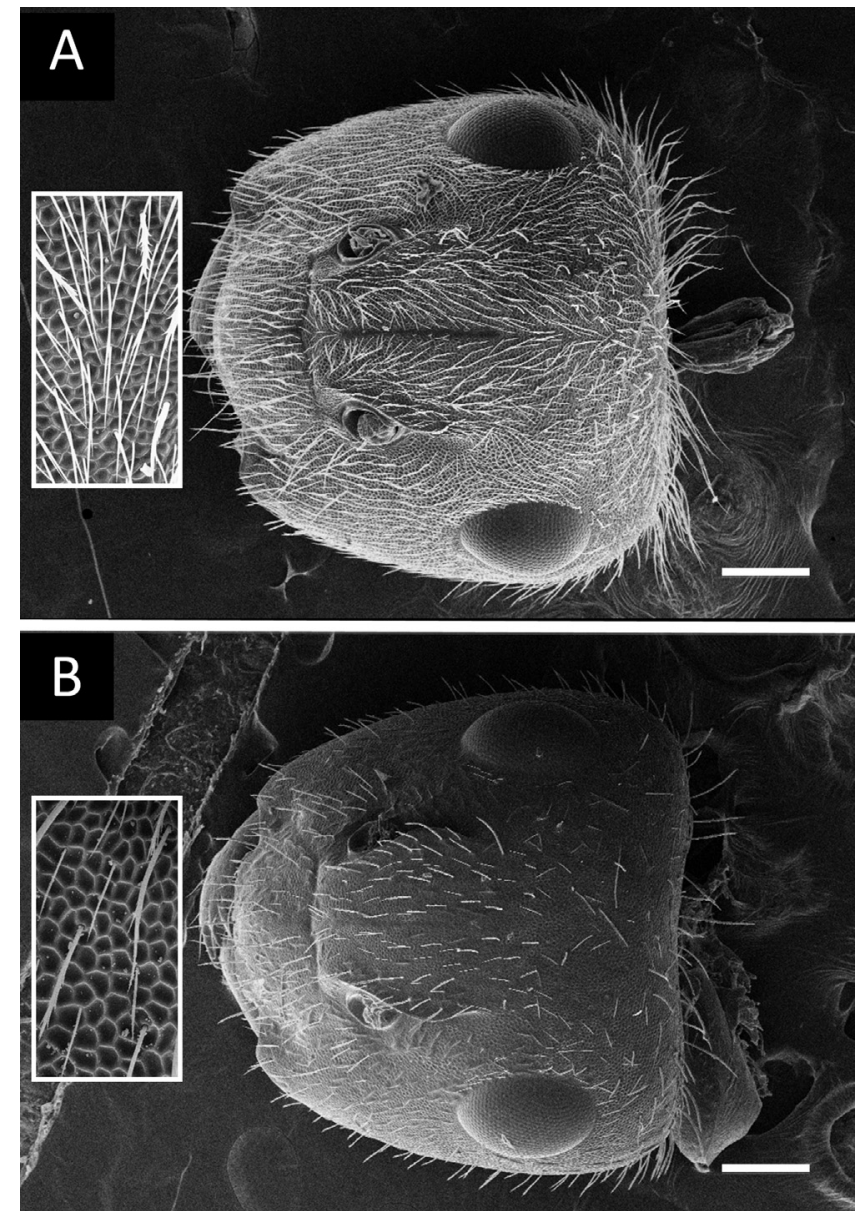

Fig. 3. Frontal view of Camponotus major workers heads. A) C. textor; B) C. senex. Insets are $100 \times 200 \mu \mathrm{m}$ close-ups on left vertex surface. Size of scale bars $(\mu \mathrm{m}): 290 ; 300$.

$12{ }^{\circ} \mathrm{C} / \mathrm{min}$ from $50{ }^{\circ} \mathrm{C}$ to $290{ }^{\circ} \mathrm{C}$ with hold pause for 6 minutes, followed by an increase of $30{ }^{\circ} \mathrm{C} / \mathrm{min}$ from $290{ }^{\circ} \mathrm{C}$ to $320^{\circ} \mathrm{C}$ followed by a final hold time of 1 min" (quoted from Fox et al., 2016 on Fox et al., 2012). Obtained chromatogram files available in Fox et al. (2017).

Compounds recovered from body washes included alkaloids and cuticular hydrocarbons which were identified by matching their retention indices and acquired mass spectra with registers in the internal mass spectra library (Wiley 275) and from comparing with published literature (Vanden Dool and Kratz, 1963; Adams, 1995; Leclercq et al., 1994, 1996; Chen and Fadamiro, 2009; Campos et al., 2012) and with accumulated chromatograms of previous analyses using the same protocol (Fox et al., 2012; EGPF unpublished results) with different ant species. Injected $n$-alkane standards (ladder range C11-C33) were used to bracket the retention indexes as whole numbers: 8000, 1200, 1500,1700 and 2700 (Adams, 1995). In order to confirm the detected the presence of piperidine alkaloids within peaks, chromatograms were masked for diagnostic ions $\mathrm{m} / \mathrm{z} 98$ and 124 by post-run single ion monitoring.

\subsection{Molecular data}

Specimens were fixed and preserved in 95\% ethanol. Total DNA purification was performed using a modified CTAB protocol (2\% CTAB, 20 mM EDTA, 0.1 M Tris, $1.4 \mathrm{mM} \mathrm{NaCl}$; Lazoski et al., 2011), followed by precipitation induced by sodium acetate and isopropanol. Polymerase chain reactions (PCR) were set up using 1 unit of Taq polymerase (GE Life Sciences), $0.2 \mathrm{mM}$ of each dNTP, $0.3 \mu \mathrm{M}$ of each primer, $2.5 \mathrm{mM}$ of $\mathrm{MgCl}_{2}, 2.5 \mu \mathrm{L}$ of PCR buffer (10X) and $1 \mu \mathrm{L}$ of DNA (10 ng) as a template in a final volume of $25 \mu \mathrm{L}$. The thermo cycling conditions were one initial cycle of $3 \mathrm{~min}$ at $95^{\circ} \mathrm{C}$, which was followed by 30 cycles consisting of the following steps: $94^{\circ} \mathrm{C}$ for $1 \mathrm{~min}, 50{ }^{\circ} \mathrm{C}$ for $1 \mathrm{~min}$, and $72{ }^{\circ} \mathrm{C}$ for $1 \mathrm{~min}$, and a final extension step at $72{ }^{\circ} \mathrm{C}$ for $5 \mathrm{~min}$. Negative controls, involving template-free reactions, were included in all PCR amplifications. A portion of the mitochondrial region was amplified using primers: L-CO1490 (GCA ACG ATG ATT TTT CTC) and H-CO2198 (GCC TTT TGG GGC CTT GGG) for cytochrome c oxidase I (COI) (Folmer et al., 1994). A portion of the first internal transcribed ribosomal spacer (ITS-1) was amplified using primers: SP-1-5' (CAC ACC GCC CGT CGC TAC TA) and SP-1-3' (ATT TAG CTG CGG TCT TCA TC) (Chu et al., 2001). Both strands of PCR products were purified with a GFX ${ }^{\mathrm{TM}}$ PCR DNA and Gel Band Purification Kit (GE Life Sciences), according to the manufacturer's instructions, and sequenced in an ABI 3500 automatic sequencer with the same sets of primers used for the PCR reaction. All sequences obtained were deposited in GenBank nos. KY349927-KY349932. Additionally, Camponotus sequences from the GenBank were selected for inclusion in our phylogenetic analyses (Accession Numbers as listed in Fig. 9 and pertaining Supplementary Information in Fox et al. (2017)) largely based on the choices for outgroup and most reliable deposits by Clouse et al. (2015) and sensible agreement with biogeographical data from Antwiki.org, for further details see dataset at Fox et al. (2017).

The obtained DNA sequences were edited with SEQMAN 7.0 (DNASTAR Inc.) and aligned with the CLUSTALW (Thompson et al., 1994) algorithm present on MEGA 5.0 (Tamura et al., 2011). Estimates of sequence polymorphism were obtained through DNASP 5 (Librado and Rozas, 2009). The maximum likelihood algorithm of MEGA 5.0 was used to obtain the best nucleotide substitution model through the
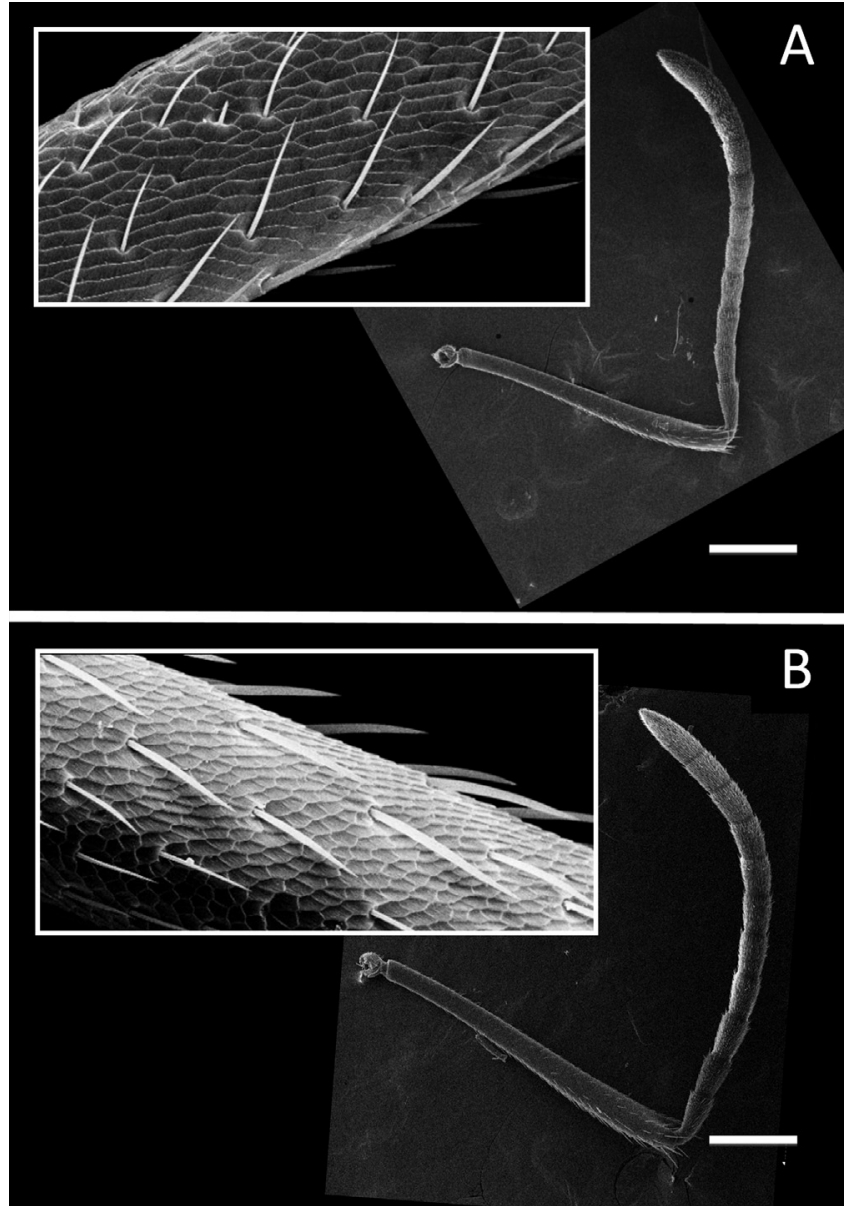

Fig. 4. Antennae and closer view of medial antennal scape bristles on: A) Camponotus textor; B) C. senex. Insets are $100 \times 200 \mu \mathrm{m}$ close-ups on antennal scape surface. Size of scale bars $(\mu \mathrm{m}): 325 ; 385$. 

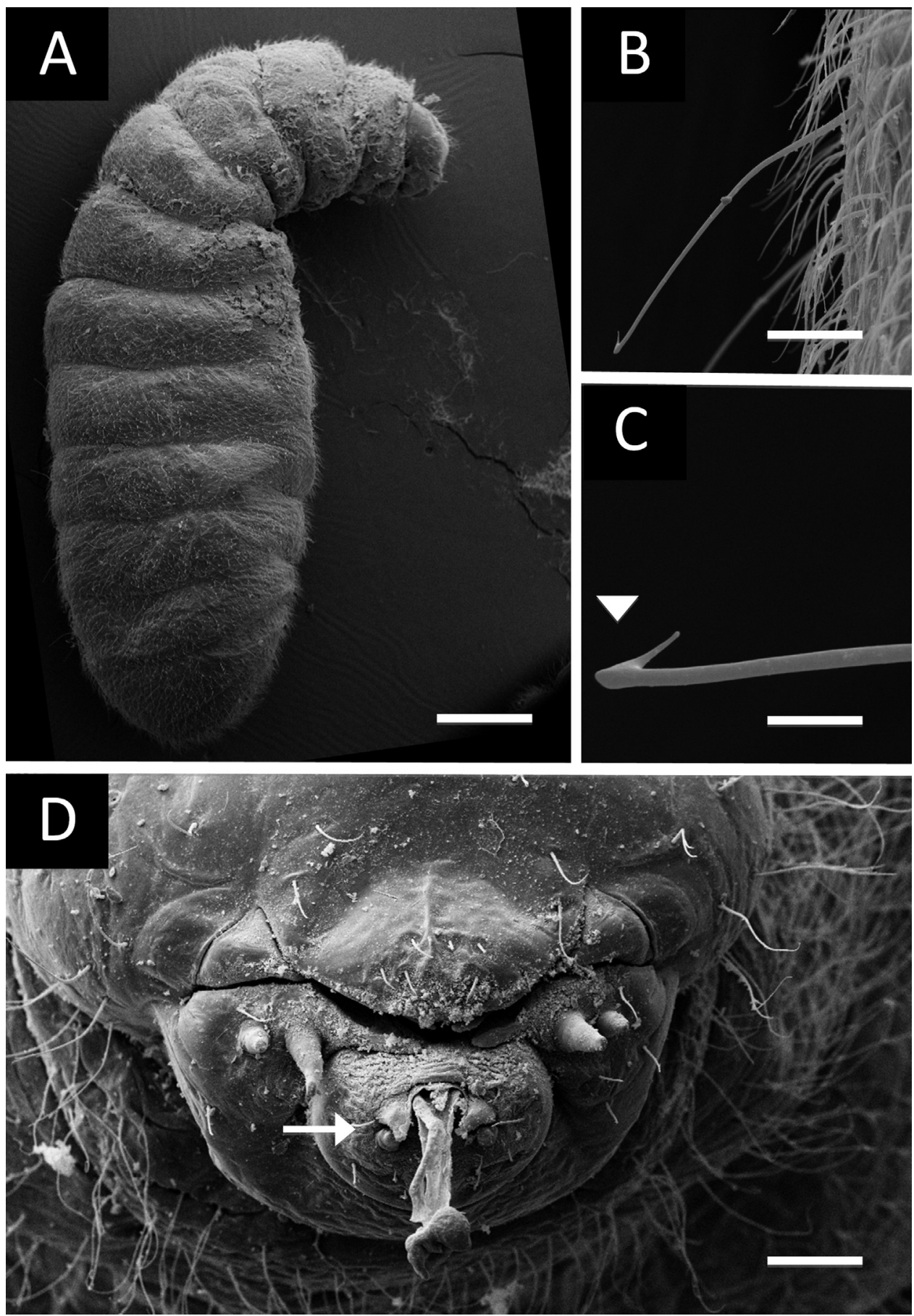

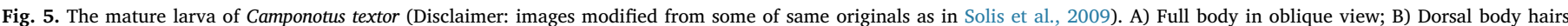

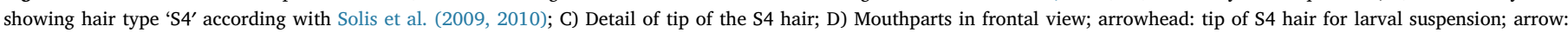
spinneret. Size of scale bars $(\mu \mathrm{m})$ : 500, 30, 8, 60 .

estimation of the lowest Bayesian Information Criterion (BIC) score. All analyses were done using the nucleotide evolution model available that closely resembled the one indicated by MEGA 5.0. For the COI dataset, the HKY model (Hasegawa et al., 1985) with estimates of invariable sites (I) and gamma distribution (G) was used. The Maximum Likelihood analysis was performed with the program PHYML (Guindon and Gascuel, 2003) using default parameters and 1000 Bootstrap branch support replicates.

\subsection{Data analysis}

Colourful pictures of mounted specimens where assembled for composite images with Photoshop CS 2006; all original images provided as Supplementary Materials (dataset available as Fox et al., 2017). Chromatograms were filtered and prepared with PostRun Data Analysis Shimadzu 2010; original chromatogram files used provided as Supplementary Materials (Fox et al., 2017). All final images were assembled and exported with PowerPoint 2007 adjusted for increased resolution, and edited strictly for minor adjustments in cropping margins and orientation; all originals provided as Supplementary Materials (Fox et al., 2017). 

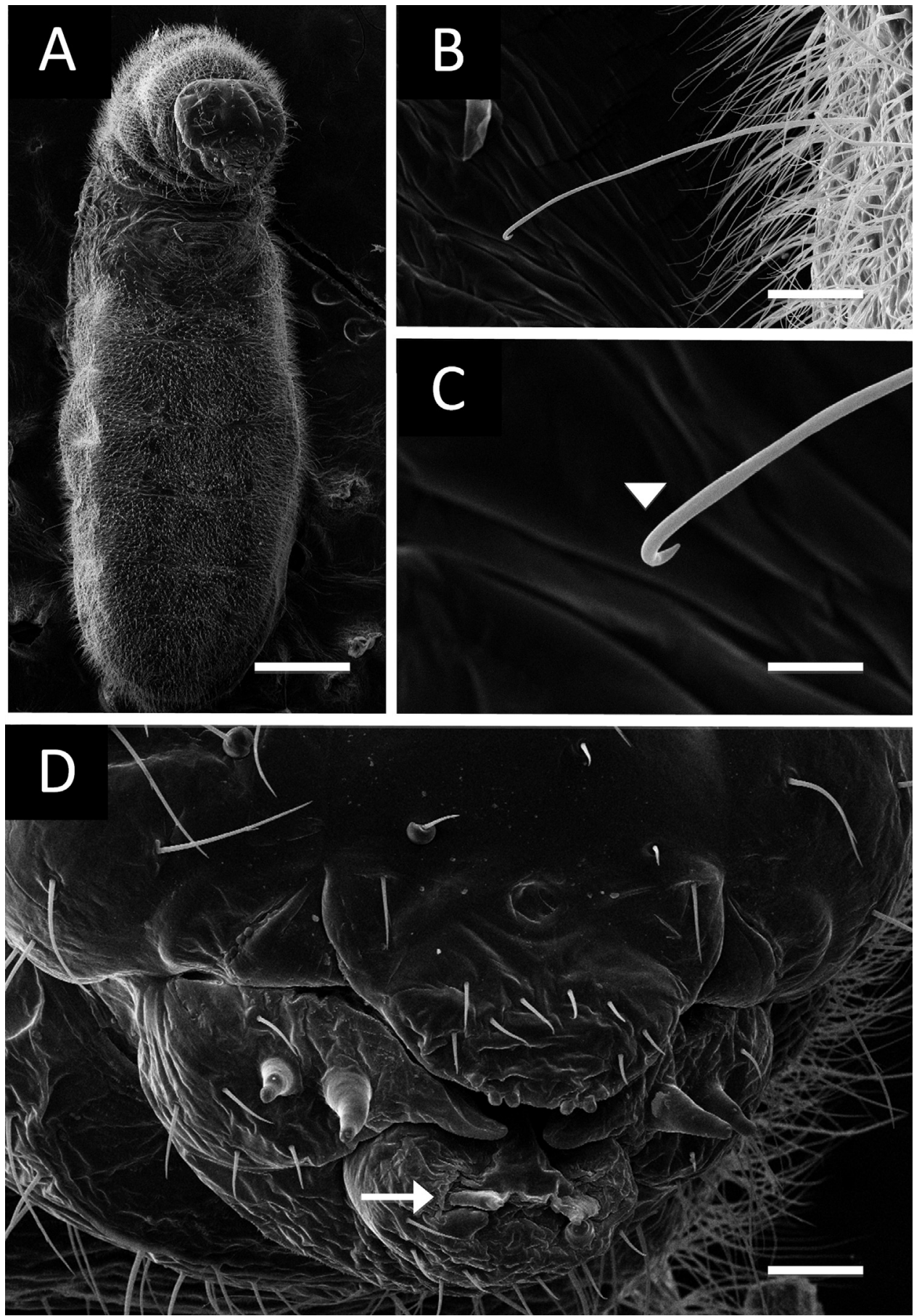

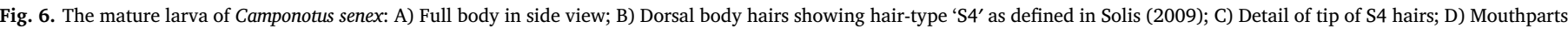
in frontal view; arrowhead: tip of S4 hair; arrow: spinneret. Size of scale bars $(\mu \mathrm{m}): 470,50,5,50$.

\section{Results}

\subsection{Adult worker morphology}

General appearance: Adult major worker specimens of $C$. textor (Fig. 1) differ from those C. senex (Fig. 2 ) in terms of bodily proportions and colour. There is considerable overlap in the size ranges based on the several collected specimens from both species and including the original museum types: for most specimens $C$. senex is clearly largest and bulkier as illustrated by the following obtained size ranges. Obtained head lengths range for $C$. senex was $1.70-2.38 \mathrm{~mm}$, while for $C$. textor was $1.44-1.90 \mathrm{~mm}$. The scape length range within $C$. senex was $1.70-1.90 \mathrm{~mm}$, while for $C$. textor was $1.26-1.72 \mathrm{~mm}$. Eye length for $C$. senex was $0.50-0.65 \mathrm{~mm}$ whereas for $C$. textor it was $0.44-0.51 \mathrm{~mm}$. Workers of $C$. textor look considerably smaller and more fragile than workers of $C$. senex, and their legs are distinctively more reddish, resulting in a striking contrast with the overall body colour (a pattern roughly similar to another species which is more common in Brazil, Camponotus rufipes F.). The heads of workers of $C$. senex, minor workers included, are always notably larger in relation to body size than heads of $C$. textor (compare Figs. 1 and 2). The antennal scapes of our specimens of $C$. textor were less setose than the specimens from Costa Rica illustrated in Longino (2006). In addition to these characters, the sculpture on the gaster is much rougher and granulated in deposited specimens of workers, gynes, and males of $C$. 
Table 1

Retention times and peak parameters by GC-MS of cuticular hydrocarbons recovered from whole body washes of Camponotus senex. Bold letters indicate majoritarian peaks.

\begin{tabular}{llllll}
\hline Peak no. & Retention (mins) & \% Area & \% Height & A/H & Compound Name \\
\hline 1 & 18.08 & 5.03 & 7.29 & 1.71 & C19 \\
2 & 18.91 & 1.19 & 1.73 & 3.91 & C23:0 \\
3 & 19.68 & $\mathbf{3 0 . 0 0}$ & $\mathbf{3 9 . 7 9}$ & 6.75 & $\mathbf{1 2 M e - C 2 4}$ \\
4 & 21.16 & 3.08 & 4.19 & 1.79 & C25 \\
5 & 27.44 & 4.38 & 3.66 & 1.89 & C26 \\
6 & 30.00 & 8.37 & 4.09 & 1.99 & C27 \\
7 & 29.57 & 6.24 & 6.25 & 3.49 & $13 \mathrm{Me}-\mathrm{C} 27$ \\
8 & 30.37 & $\mathbf{1 2 . 6 5}$ & $\mathbf{1 1 . 5 5}$ & 2.13 & DiMe-C27 \\
9 & 30.58 & 14.00 & 12.98 & 1.98 & C28 \\
10 & 31.13 & 15.06 & 8.47 & 0.41 & $13 \mathrm{Me}-\mathrm{C} 28$ \\
- & - & 100 & 100 & - & - \\
\hline
\end{tabular}

$\mathrm{A}=$ peak area; $\mathrm{H}=$ peak height, in bold are predominant compounds useful for species diagnosis.

Table 2

Retention times and peak parameters by GC-MS of cuticular hydrocarbons recovered from whole body washes of Camponotus textor. Bold letters indicate majoritarian peaks.

\begin{tabular}{|c|c|c|c|c|c|}
\hline Peak no. & Retention (mins) & $\%$ Area & \% Height & $\mathrm{A} / \mathrm{H}$ & Compound Name \\
\hline 1 & 18.07 & 1.30 & 2.49 & 1.76 & C19 \\
\hline 2 & 21.49 & 2.57 & 1.11 & 7.85 & C23:0 \\
\hline 3 & 22.11 & 4.53 & 1.69 & 9.09 & $12 \mathrm{Me}-\mathrm{C} 24$ \\
\hline 4 & 22.50 & 0.80 & 1.29 & 2.11 & $\mathrm{C} 25$ \\
\hline 5 & 23.14 & 2.58 & 2.38 & 3.69 & $\mathrm{C} 26$ \\
\hline 6 & 23.48 & 2.44 & 1.31 & 6.23 & Und \\
\hline 7 & 23.68 & 3.53 & 2.56 & 4.70 & Und \\
\hline 8 & 23.82 & 6.12 & 11.10 & 1.88 & C27 \\
\hline 9 & 24.05 & 2.52 & 2.21 & 3.87 & $13 \mathrm{Me}-\mathrm{C} 27$ \\
\hline 10 & 24.37 & 5.31 & 8.42 & 2.14 & DiMet-C27 \\
\hline 11 & 24.57 & 2.99 & 5.24 & 1.93 & $\mathrm{C} 28$ \\
\hline 12 & 25.10 & 1.42 & 1.93 & 2.50 & 7Me-C28 \\
\hline 13 & 25.44 & 7.39 & 10.47 & 2.40 & C29 \\
\hline 14 & 25.73 & 5.87 & 4.67 & 4.28 & 9Me-C29 \\
\hline 15 & 26.05 & 3.17 & 4.39 & 2.43 & Und \\
\hline 16 & 26.18 & 3.66 & 6.24 & 2.01 & C30 \\
\hline 17 & 26.45 & 2.14 & 2.27 & 3.19 & C31 \\
\hline 18 & 26.79 & 2.05 & 1.28 & 5.44 & 5Me-C31 \\
\hline 19 & 27.16 & 1.07 & 0.87 & 4.18 & DiMe-C31 \\
\hline 20 & 27.66 & 2.68 & 2.94 & 3.10 & C32 \\
\hline 21 & 28.06 & 9.00 & 4.60 & 6.68 & $15 \mathrm{Me}-\mathrm{C} 32$ \\
\hline 22 & 28.51 & 6.17 & 2.59 & 8.07 & DiMe-C32 \\
\hline 23 & 29.54 & 1.75 & 1.15 & 5.17 & Und \\
\hline 24 & 29.77 & 2.98 & 2.62 & 3.85 & Und \\
\hline 25 & 30.36 & 3.30 & 3.39 & 3.30 & C33 \\
\hline 26 & 30.55 & 4.39 & 2.10 & 7.06 & Und \\
\hline 27 & 31.20 & 1.58 & 1.20 & 4.43 & C34:0 \\
\hline- & - & 100 & 100 & - & - \\
\hline
\end{tabular}

Und - hydrocarbons of undetermined identity, bold markings indicate the predominant compounds useful in species diagnosis.

textor than in the equivalent castes of $C$. senex (not shown). Under high light magnification the individual units of the dorsum of the gaster can be seen as separated by a series of crossed ridges. The similar surface of the gaster of $C$. senex is somewhat smoother and more polished, with the individual units fusing into each other (see insets on Fig. 1 and Fig. 2).

\subsection{Larval morphology}

The mature larvae of $C$. senex (Fig. 5) were compared with data from C. textor as obtained in Solis et al. (2009) (represented on Fig. 6), where a single conspicuous difference was observed: the presence of dorsal hooked body hair referred to as hair type "S4" (Solis et al., 2009, 2010). In short, it is an unbranched, long hair apparently used for hanging the larva, and the homologous S4-type hairs the two species present tip modifications: the tip is hooked in C. senex (Fig. 5B, C) but sharply angulate like a pick in C. textor (Fig. 6B, C). The S4-type hairs are found organised in rows towards the dorsum of each body somite in both species, in an homologous pattern which is easily observable under a light microscope; the difference at the tip becomes evident only upon higher magnification observation of mounted specimens (Fig. 6B, C). Otherwise, larval shape and general pattern of hair distribution (Figs. $5 \mathrm{~A}, 6 \mathrm{~A}$ ), and details of external mouthparts (Figs. $5 \mathrm{D}, 6 \mathrm{D}$ ) were equivalent, including the head width intervals of larvae with and without pseudopalps (not shown) thus suggesting the number of larval instars in both species and relative sizes should be the same.

\subsection{Cuticular hydrocarbons}

The use of molecular markers and chemical tools is a proposed strategy in such cases of overlapping morphology (Seifert, 2009; Fox et al., 2012; Ronque et al., 2015). The main cuticular hydrocarbons identified for both species are listed on Tables 1 and 2, based on the results of chromatograms as exemplified in Figs. 7 and 8. The main cuticular hydrocarbons for both species were C29 and 15Me-C29, however followed by $9 \mathrm{Me}-\mathrm{C} 29$ in C. senex and C27 in C. textor. Also trace amounts of solenopsin alkaloids (Figs. 5 B, 6 B) of same retention time and fragmentation spectra as solenopsin A (detected in $C$. senex) and solenopsin B (detected in both) and solenopsin C in C. textor were detected by masking for specific ions.

\subsection{Molecular data}

Five individual workers from each colony were used, totalling 20 obtained sequences for each marker. From the ITS-1 marker only one haplotype was found for each Camponotus species (Table 3). The mitochondrial COI gene proved more polymorphic (Table 3), yielding two haplotypes for each species. Both mitochondrial (COI) and nuclear (ITS-1) suggest the two species are not the result of a recent speciation. While intraspecific genetic divergences (K2P) were low $(0.000-0.005$ for C. textor and 0.000-0.006 for $C$. senex), divergence was high between $C$. textor and C. senex (K2P for COI: 0.140-0.148; K2P for ITS-1: 0.035). These degrees of interspecific genetic differentiation were herein observed among the other analysed Camponotus species (COI: 0.128-0.261; ITS-1: 0.091-0.107). Noting a close relatedness according with relative distance in ITS, the obtained sequences demonstrate these species are sister species relative to the Camponotus sequences available online (Fig. 9), however possibly not sister species in reality. Only further sampling efforts of more closely related taxa can add resolution enabling to determine how close these species in fact are. A separate tree was generated including the sequences of Ramalho et al. (2016a) but is herein provided in $\mathrm{S} 1$ as the sequence comparison range was much shorter (ca. $45 \%$ of usable overlap) because of different primers, thus generating herein a less stable phylogeny.

\subsection{Differences in defensive behaviour}

During collections we observed aspects of the ants' reaction when disturbed. Workers of $C$. textor are much more aggressive when their nest is disturbed and will attack in great numbers while making a distinctive buzzing sound from inside the nest, yet their bites are not painful as they apparently do not apply venom while biting. On the other hand, C. senex is shy when their poorly-structured nests are exposed, running rapidly to escape (renders collecting their brood difficult in the field), yet will inflict a painful bite with acid venom application if handled without care.

\section{Discussion and conclusions}

\subsection{Morphology}

4.1.1. Adults

Based only on the general relative sizes and colours, the two species 

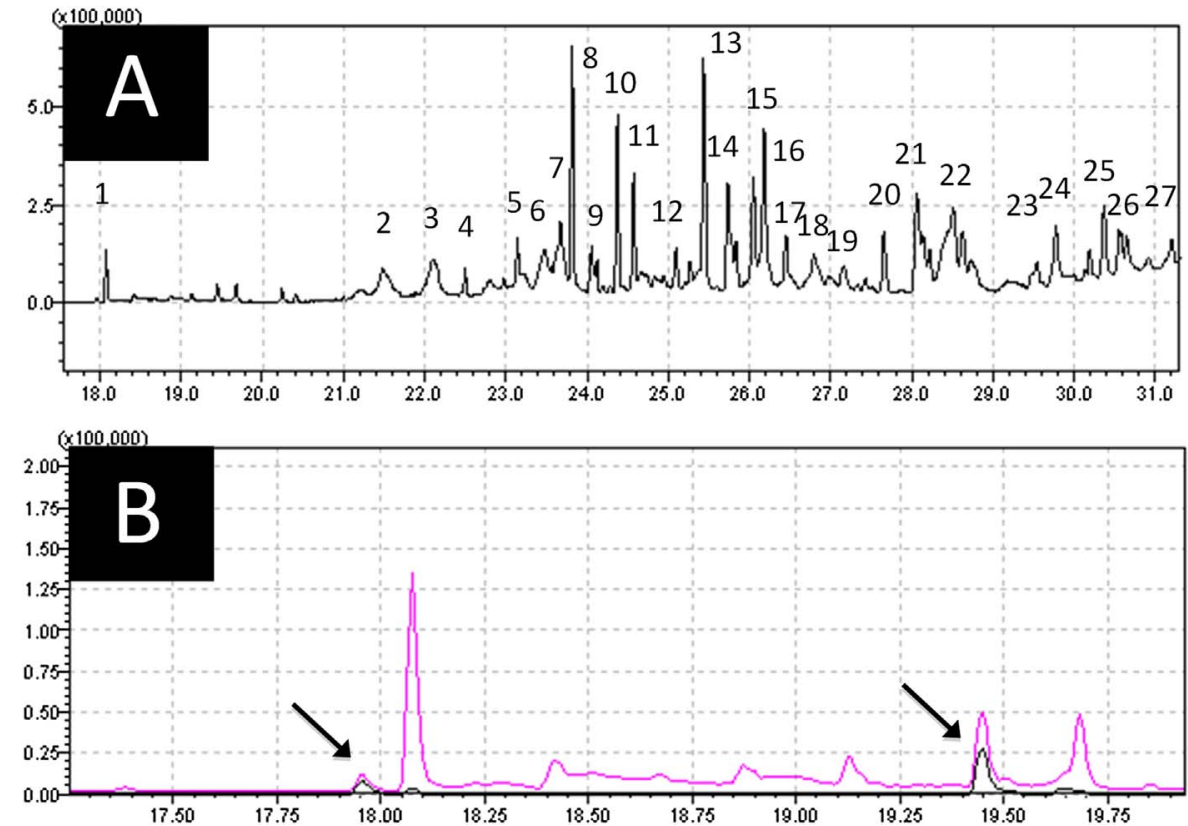

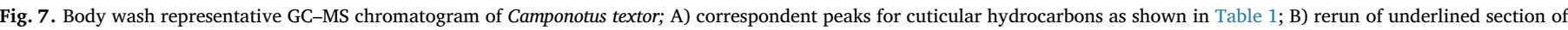

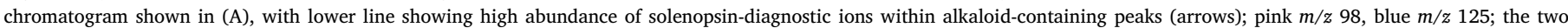

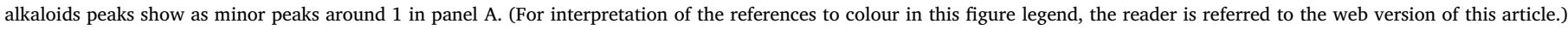
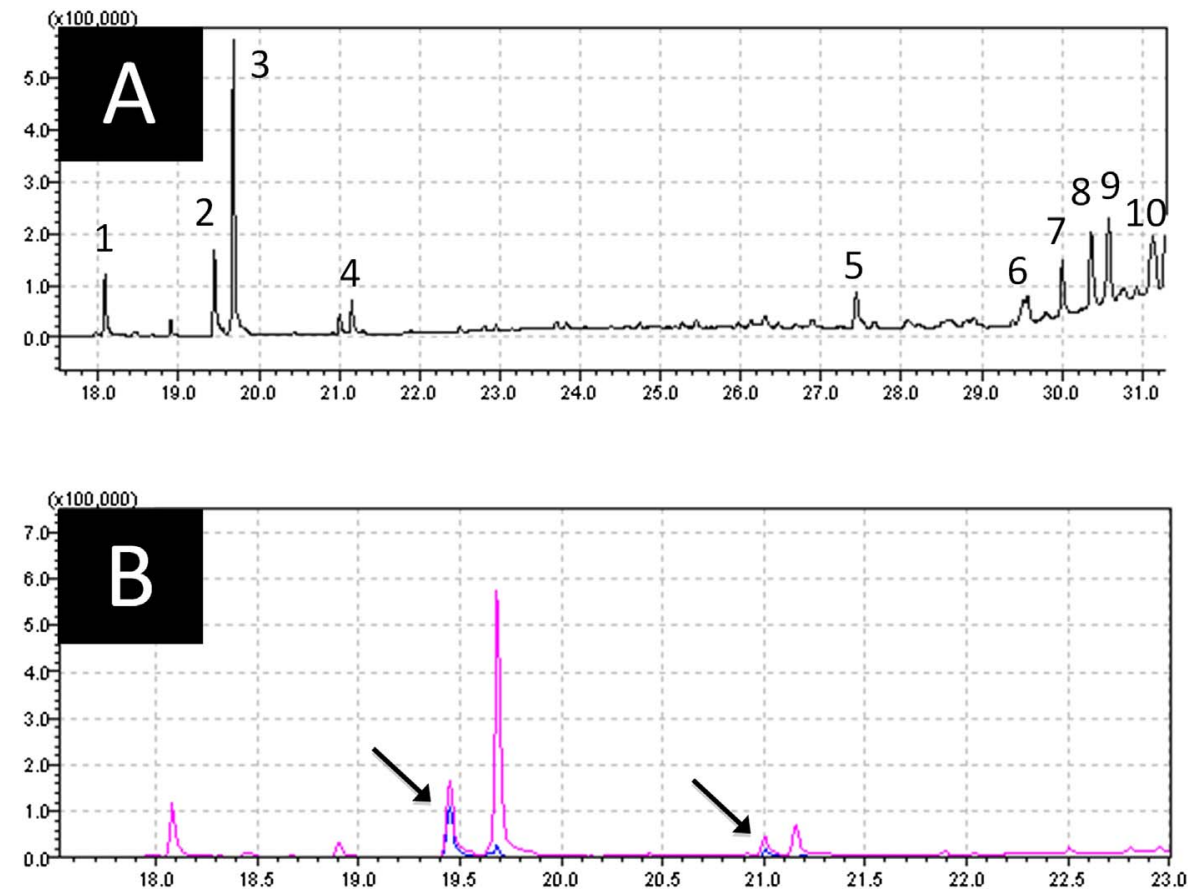

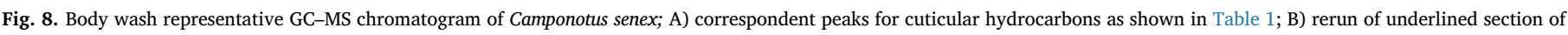

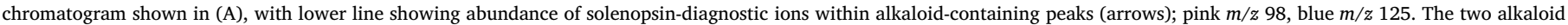

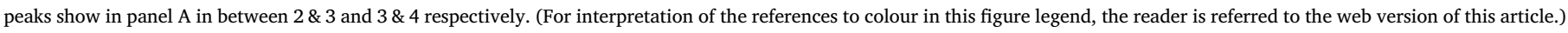

can be differentiated when compared to each other. The obtained measured limits for head, eyes, and antenna illustrate how $C$. senex is generally larger than $C$. textor and provide margins for species identification however only reliable where there is no overlap. To that respect, there was little overlap in eye length limits, meaning this is the most useful head structure for species diagnosis based on relative sizes. Clear differences in colour and relative sizes also seem evident in the specimens analysed by Longino (2006, Pictures 4-7). The morphology of adults suggests the species can be separated at first sight by colour and bulk, but such plastic characters should always be considered with caution, as discrete variations in size and colour patterns are prone to environmental factors (e.g. climate, colony nutrition) or even alteration of specimens by fixation (e.g. observed in a similar case by Ronque et al., 2015). Given that perceived differences between adults of the two species include variations in bodily proportions, we propose a phenetics study based on extensive sample analysis should provide simpler reliable characters for species separation, namely the application of the NUMOBAT techniques as described by Seifert (2009). In fact the observations by Perez-Lachaud and Lachaud (2014) and Longino (2006) strongly suggest the existence of further cryptic species, which 
warrant a dedicated study with NUMOBAT analyses of the available museum specimens. For the moment, considering the inclusion of type specimens the proposed measure limits should suffice to separate among "typical" specimens throughout the Neotropical Region, but there is still considerable overlap which gives room for uncertainty. Some variants of $C$. textor were reported by other authors to be easily confused with $C$. senex, which led to the proposal of diagnostic characters based on hair distribution as described below.

The current diagnostic characters to separate workers of $C$. senex from $C$. textor are based on the form and abundance of setae on the antennal scape and gaster (Longino, 2006; Mackay, 2014), which should be less abundant in $C$. senex than in $C$. textor. A high density of "appressed hairs" on the gaster of $C$. textor will usually completely obscure the surface by light microscopy, but such differences can be subtle and depend on the examination of several specimens in a series. From comparing images of these structures (Figs. 1-4) by SEM the differences in body setae types, distribution, and density become clear. Such setae called the "appressed hairs" are in fact finer, shorter setae that run parallel to the surface. In fact our microanalysis demonstrates such hairs will typically abound on the various body parts of $C$. textor, mainly on the gaster (Figs. 1 B and 2 B), the head (Fig. 3), and the mesosoma (not shown), but not as distinct on the antennae (Fig. 4). Regarding the antennae of $C$. textor, they are reported to typically lack suberect or erect hairs on the scape, however specimens from Brazil will occasionally have seemingly suberect hairs on the scape rendering separation more difficult (Fig. 4).

However, inconspicuous differences such as the number and morphology of body setae can be hard to ascertain by the inexperienced observer. Based on ultrastructural images (Figs. 1-4) of these setal distribution and density, we conclude the presence and abundance of appressed gastral setae remains as the most convenient and reliable distinguishing character, particularly on the gaster. The abundant appressed hairs (i.e. shorter, low setae) on the gaster of $C$. textor will obscure the surface and give it a golden hue, however often such pattern can be subtle under the microscope and depend on the examination of several specimens in a series to make sure. Our images demonstrate the number of setae on gaster of $C$. textor may be more than double those on $C$. senex (compare insets Figs. 1 and 2). The setae on the antennal scapes are not as helpful as we originally thought (Mackay, 2014) as the presence of "suberect hairs" proved too variable, and the term is poorly defined. Fig. 4 illustrates suberect is a misleading term given the antennal scape hairs of both Camponotus seem suberect in higher magnification. In addition to these characters we herein propose the utility of differences in sculpture, particularly on the gaster. These different patterns can be seen with difficulty in a light microscope, but are more obvious in the scanning electron micrographs in Figs. $1 \mathrm{~B}$ and $2 \mathrm{~B}$, where one can see the differences in depth between the surface hexagonal ridges.

\subsubsection{Larvae}

Larval characters are naturally less practical as samples may be harder to obtain yet useful as clear difference can be observed at the dorsum of mature larvae under high magnification, at the tip of a conspicuously long hairs named 'subtype S4' in previous publications (see Solis et al., 2009, 2010 for details on hair terminology). The biological significance of these morphological adaptations is an interesting aspect to be further investigated, but it is tempting to assume these hairs are either employed in hanging larvae or in securing prepupae to their cocoons. It is interesting to note both species appear to have the same number of larval instars, based on head width similarities, as considerable intrageneric variation in the number of instars is reported in Camponotus. (Solis et al. (2010)).

While SEM analysis proves very useful for the observation and documentation of such details, this expensive microscope is not readily available for most taxonomists and observation depends on special sample preservation and preparation apart from technical experience. Moreover, observation of specific minute structures typically requires
Table 3

DNA polymorphism of Camponotus textor and C. senex using mitochondrial and nuclear markers.

\begin{tabular}{llllll}
\hline & \multicolumn{1}{l}{ COI } & & & ITS-1 & \\
\cline { 2 - 3 } \cline { 5 - 6 } & C. textor & C. senex & & C. textor & C. senex \\
\hline Length (bp) & 658 & 658 & & 1029 & 1019 \\
$\mathrm{~N}_{\text {seq }}$ & 10 & 10 & & 8 & 10 \\
$\mathrm{~N}_{\text {hap }}$ & 2 & 2 & & 1 & 1 \\
C:T:A:G & $36: 24: 28: 12$ & $37: 22: 29: 12$ & & $20: 27: 23: 30$ & $20: 27: 22: 31$ \\
$\mathrm{~S}$ & 3 & 4 & & - & - \\
$\mathrm{P}$ & 3 & 4 & & - & - \\
$\mathrm{h}$ & 0.5556 & 0.5556 & & - & - \\
$\pi$ & 0.0025 & 0.0034 & & - & - \\
\hline
\end{tabular}

$\mathrm{N}_{\text {seq }}$ : Number of sequences; $\mathrm{N}_{\text {hap }}$ : Number of haplotypes; C:T:A:G: Nucleotide composition; S: Variable sites; P: Parsimony informative sites; h: Haplotype diversity; $\pi$ : Nucleotide diversity.

special expertise and excellent sample preservation of several specimens. A practical and non-destructive alternative is the application of phenetics as proposed above.

Finally while the two species are sympatric over most of their extensive ranges and in our study area, we saw no morphological indication of hybridization, further supporting the hypothesis that they are separate species.

\subsection{Chemical characters}

Both the amount and diversity of cuticular hydrocarbons (CHCs) were more pronounced in $C$. textor than in $C$. senex. The range for intraspecific variation of CHCs for $C$. textor was previously described by Campos et al. (2012). Our results with all injected samples generally confirm the pattern shown by Campos et al. (2012) on the diversity of compounds and identified classes, however their specific identifications of compounds were strikingly different. We were unfortunately unsuccessful in trying to obtain the original chromatograms from the authors, and Campos et al. (2012) does not state exactly how each different alkane was assigned on their chromatograms. Given that hydrocarbon MS fragmentation spectra are typically similar within classes and their molecular ions will frequently not appear in crude extracts, the identification of hydrocarbon species is often controversial. We observe our obtained CHCs profile are compatible with their results for major workers if one assumes a mistake in their original estimation of carbon chain length: e.g. where $\mathrm{C} 28$ followed by MeC28derived compounds are corresponding to our observed C29 derivatives shown in Tables 1 and 2. Such a pattern is also in agreement with the fact that specimens where obtained from the same geographical region, thus probably same population.

Regarding the two species, their obtained chromatograms were quite different, and the pattern for $C$. senex is very distant from the observed intraspecific variation described by Campos et al. (2012) for C. textor. This illustrates CHCs can be reliably employed to separate these specimens, where possible.

Finally, probable alkaloids detected in the body wash extracts presented the same retention times and spectra as venom alkaloids recovered from Solenopsis spp. fire ants (Fox et al., 2012) however in trace amounts. Although this is the first report of alkaloids from body extracts of Formicinae ants, such compounds may have come from field contamination, considering these species are sympatric to and potentially compete with alkaloid-abundant fire ants.

\subsection{Molecular characters}

In agreement with the differences in biology and CHCs, the genetic divergence estimates of KP2 of both mitochondrial and nuclear genes suggest that the two species are not immediately related, but well 


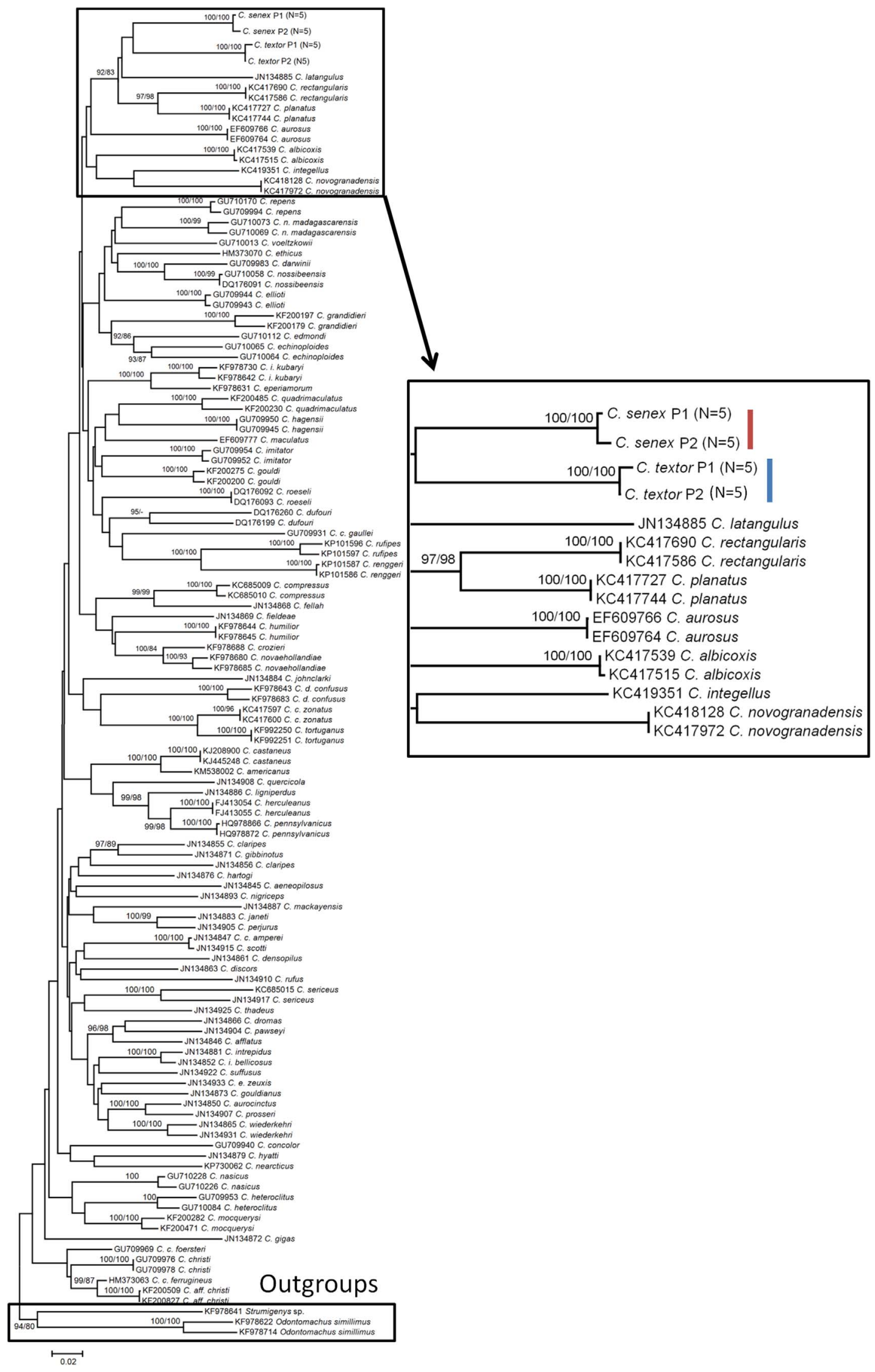

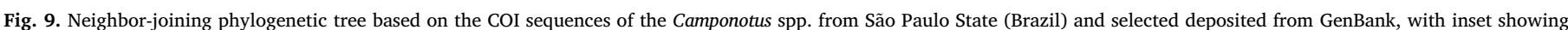

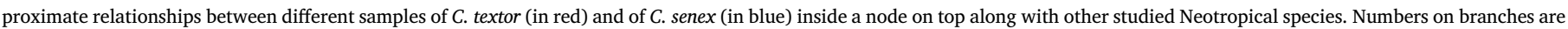

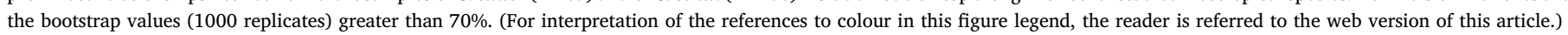




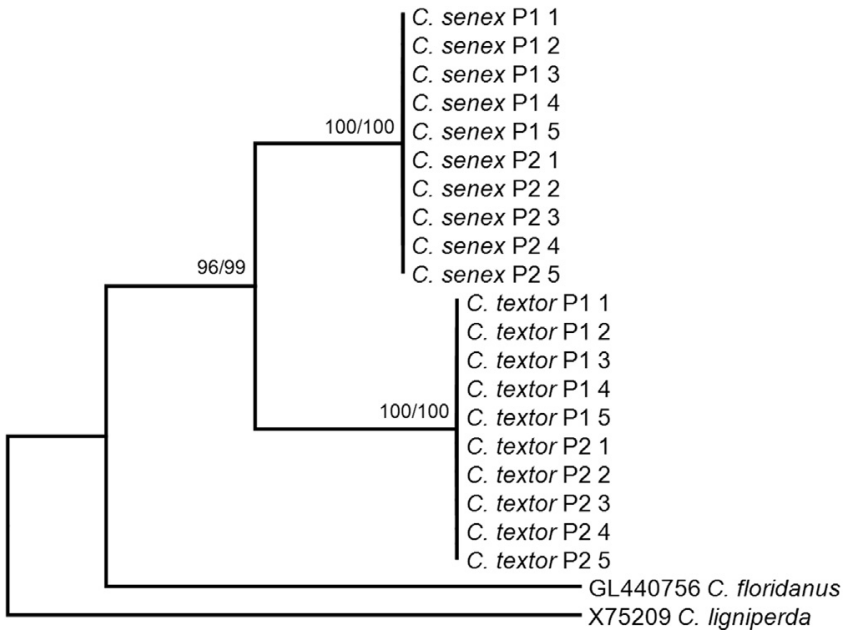

0.005

Fig. 10. Neighbor-joining phylogenetic tree based on obtained ITS-1 sequences of Camponotus senex and C. textor from Southeast Brazil with GenBank C. floridanus and C. ligniperda as outgroups. Numbers on branches are the bootstrap values (1000 replicates) greater than $90 \%$

separated taxa. Whilst the phylogeny on Fig. 9 supported by Fig. 10 place $C$. senex and $C$. textor in the same branch as relative to other available Camponotus species, the obtained distances seem relatively high as to consider them sister species. Based on the limited amount of information on ITS-1 it was observed that factually sister species of Melipona had $1.4 \%$ divergence in ITS-1 (Fernandes-Salomão et al., 2005); concerning COI the distance estimated for congeneric species within Hymenoptera was once estimated as $11 \pm 3.8$ (sd)\% from a major scale analysis by Herbert et al., (2003). Thus the fact that online available species used in constructing Fig. 9 are not directly sister species, the obtained KP2 diverge estimates suggest $C$. senex and $C$. textor are not closely related as the result of a recent speciation event, which is consistent with the observed differences in behaviour and CHCs. The considerable distance between these species was recently estimated in an independent investigation by Ramalho et al. (2016a). We have also built a tree incorporating the sequences deposited by Ramalho et al. (2016a), but found limited compatibility with our primer results as the overlap was much shorter (see supplementary S1). Taken together, the present molecular evidence supports Ramalho et al. (2016a) reinforcing the remarkable fact observed by Longino (2006) that "the construction of silk nests and the weak to absent polymorphism have evolved independently in different lineages", on complex traits evolving multiple times within the same lineage. It should be kept in mind that often particularities such as worker polymorphism and life habits were taken as arguments for grouping generally similar species into assemblages such as subgenera. It should be noted however that while biogeographical distribution is strongly convergent with genetic evidence within Camponotus, we observed no significant overlap in phylogeny supporting the current use of subgenera within Camponotus; e.g. C. textor is not closely related with other weakly polymorphic species nor do they seem to be closely related among themselves (Fig. 9).

Finally, in the context of molecular biology, we should emphasize that the recent study by Ramalho et al. (2016b) found evidence for intraspecific cryptic populations within the nominal C. textor by $28 \mathrm{~S}$ rDNA which probably correspond to those noted by previous authors (Longino, 2006; Lachaud and Lachaud, 2014). We herein did not find such evidence (Fig. 10, Table 3) but our samples probably belonged all in the same population. We believe multidisciplinary studies searching for the traits described herein and genes in Ramalho et al. (2016a,b) should be applied to these more separated populations of $C$. textor to test for cryptic taxa.

\subsection{Differences in defensive behaviour}

Concerning their markedly different behaviour, both in nest structure and defense, we believe field notes can already provide useful information for a later species diagnosis. Such patterns are easily applied in conjunction with previous observations of specific nesting habits (Longino, 2006; Ramalho et al., 2016a) and habitat preferences (Ronque et al., 2015).

\section{Final considerations}

In conclusion, the present multidisciplinary study increases the body of evidence supporting the separation of $C$. textor as a distinct species to $C$. senex, while clarifying on their degrees of phylogenetic relationship. We believe some of the additional evidence will facilitate species diagnosis (the most reliable updated characters being eye length, gaster hairs, and cuticular hydrocarbons) and point to strategies to be adopted in future investigations. Given that ant taxa typically abound with cryptic species (Seifert, 2009), we feel additional multidisciplinary studies are necessary for refining myrmecology taxonomy and such studies are still too few (e.g. Lucas et al., 2002) given the dimension of the issue as discussed by Seifert (2009).

\section{Acknowledgements}

We would like to thank the Ernst Mayr Fund of the Museum of Comparative Zoology at Harvard University for supporting our visits to examine the extensive series of both species, Stefan Cover for allowing access to the Ant Room, and Dr. Gary Alpert for providing lodging. The work was also supported by a generous donation from the estate of Maxie Groce Templeton. The termite species was kindly identified by Eliana Marques Cancello (MZUSP, São Paulo, Brazil). Georgia C. Atella provided access to the gas-chromatography equipment used where useful technical assistance was provided by Mileane de Souza Busch.

Three anonymous reviewers contributed with useful comments.

\section{References}

Adams, R.P., 1995. Identification of Essential Oil Components by Gas Chromatography/ Mass Spectroscopy. Allured Publishing Corporation, USA.

Bickford, D., Lohman, D.J., Sodhi, N.S., Ng, P.K.L., Meier, R., Winker, K., Ingram, K.K., Das, I., 2007. Cryptic species as a window on diversity and conservation. Trends Ecol. Evol. 22, 148-155.

Campos, M.C.G., Campos, M.L.G., Turatti, I.C.C., Nascimento, F.S., 2012. Cuticular hydrocarbon variation of castes and sex in the weaver ant Camponotus textor (Hymenoptera: Formicidae). Sociobiology 59 (3), 1025-1036.

Chen, L., Fadamiro, H.Y., 2009. Re-investigation of venom chemistry of Solenopsis fire ants I: identification of novel alkaloids in S. richteri. Toxicon 53, 469-478.

Chu, K.H., Li, C.P., Ho, H.Y., 2001. The first internal transcribed spacer (ITS-1) of ribosomal DNA as a molecular marker for phylogenetic and population analyses in Crustacea. Mar. Biotechnol. 3, 355-361. http://dx.doi.org/10.1007/s101260010014-5.

Clouse, R.M., Janda, M., Blanchard, B., Sharma, P., Hoffmann, B.D., Andersen, A.N., Czekanski-Moir, J.E., Krushelnycky, P., Rabeling, C., Wilson, E.O., Economo, E.P., Sarnat General, E., Alpert, D.M., Wheele, W.C., 2015. Molecular phylogeny of IndoPacific carpenter ants (Hymenoptera: Formicidae, Camponotus) reveals waves of dispersal and colonization from diverse source areas. Cladistics 31, 424-437.

Fernandes-Salomão, T.M.F., Rocha, R.B., Campos, L.A.O., Araújo, E.F., 2005. The first internal transcribed spacer (ITS-1) of Melipona species (Hymenoptera: Apidae, Meliponini): characteriza tion and phylogenetic analysis. Insectes Soc. 52, 11-18.

Finlayson, T., 1967. A classification of the subfamily Pimplinae (Hymenoptera, Ichneumonidae) based on first-instar larval characteristics. Can. Entomol. 99, 2-8.

Folmer, O., Black, M., Hoeh, W., Lutz, R., Vrijenhoek, R., 1994. DNA primers for amplification of mitochondrial cytochrome c oxidase subunit I from diverse metazoan invertebrates. Mol. Mar. Biol. Biotechnol. 3, 294-299.

Fox, E.G.P., Solis, D.R., Rossi, M.L., Delabie, J.H.C., Souza, R.F., Bueno, O.C., 2012. Corrigendum to "Comparative immature morphology of Brazilian fire ants (Hymenoptera: Formicidae: Solenopsis)". Psyche 2016http://dx.doi.org/10.1155/ 2016/1013859. Article ID 1013859.

Fox, E.G.P., Mackay, W., Solis, D., Lazoski, C. [Supplementary Dataset] “Adult Major Workers and Mature Worker Larvae of Camponotus textor and Camponotus senex: Morphology, DNA, Chemical Analyses.” Mendeley Data, v.1. http://dx.doi.org/10. 17632/sc6fm9rxz8.1.

Guindon, S., Gascuel, O., 2003. A simple, fast and accurate algorithm to estimate large 
phylogenies by maximum likelihood. Syst. Biol. 52, 696-704.

Hasegawa, M., Kishino, H., Yano, T., 1985. Dating of the human ape splitting by a molecular clock of mitochondrial DNA. J. Mol. Evol. 22, 160-174.

Herbert, P.D.N., Ratnasingham, S., deWaard, J.R., 2003. Barcoding animal life: cytochrome c oxidasesubunit 1 divergences among closely related species. Proc. R. Soc. Lond. B 270.

Jones, T.H., Blum, M.S., 1982. Ant venom alkaloids from Solenopsis and Monomorium species. Tetrahedron 38, 1949-1958.

Lazoski, C., Gusmão, J., Boudry, P., Solé-Cava, A.M., 2011. Phylogeny and phylogeography of Atlantic oyster species: evolutionary history, limited genetic connectivity and isolation by distance. Mar. Ecol.-Prog. Ser. 426, 197-212.

Leclercq, S., Thirionet, I., Broeders, F., Daloze, D., Vander Meer, R., Braekman, J.C., 1994. Absolute configuration of the solenopsins, venom alkaloids of the fire ants. Tetrahedron 50, 8465-8478.

Leclercq, S., Braekman, J.C., Daloze, D., Pasteels, J.M., Vander Meer, R.K., 1996. Biosynthesis of the solenopsins, venom alkaloids of the fire ants. Naturwissenschaften 83, 222-225.

Librado, P., Rozas, J., 2009. DnaSP v5: a software for comprehensive analysis of DNA polymorphism data. Bioinformatics 25, 1451-1452.

Longino, J.T., 2006. New species and nomenclatural changes for the Costa Rican ant fauna (Hymenoptera: Formicidae). Myrmecologische Nachrichten 8, 131-143.

Lucas, C., Fresneu, D., Kolmer, K., Heinze, J., Delabie, J.H.C., Pho, D.B., 2002. A multidisciplinary approach to discriminating different taxa in the species complex Pachycondyla villosa (Formicidae). Biol. J. Linn. Soc. 75, 249-259.

Mackay, W.P., 2014. Revision of the New World Ants of the Genus. Available at www. utep.edu/leb/antgenera.htm.

Mayr, E., 1963. Animal Species and Evolution. Harvard Univ. Press, Cambridge, MA 797 pp.

Perez-Lachaud, G., Lachaud, J.P., 2014. Arboreal ant colonies as 'hot-points' of cryptic diversity for myrmecophiles: the weaver ant Camponotus sp aff. textor and its interaction network with its associates. PLoS One 9, e100155.

Pitts, J.P., Hugh, M.C.J., Ross, K.G., 2005. Cladistic analysis of the fire ants of the Solenopsis saevissima species-group (Hymenoptera: formicidae). Zool. Scr. 34, 493-505.

Ramalho, M.O., Santos, R.M., Fernandes, T.T., Morini, M.S., Bueno, O.C., 2016a.
Cytochrome c oxidase I DNA sequence of Camponotus ants with different nesting strategies is a tool for distinguishing between morphologically similar species. Genetica 144, 375-383.

Ramalho, M.O., Martins, C., Silva, L.M., Martins, V.G., Bueno, O.C., 2016b. Molecular profile of the brazilian weaver ant camponotus textor forel (Hymenoptera, formicidae). Neotrop. Entomol. 45 (5), 463-470.

Ronque, M.U., Azevedo-Silva, M., Mori, G.M., Souza, A.P., Oliveira, O.S., 2015. Three ways to distinguish species: using behavioural, ecological, and molecular data to tell apart two closely related ants, Camponotus renggeri and Camponotus rufipes (Hymenoptera: Formicidae). Zool. J. Linn. Soc. Lond. 176 (1), 170.

Schultz, T.R., Meier, R., 1995. A phylogenetic analysis of the fungus-growing ants (Hymenoptera: formicidae: Attini) based on morphological characters of the larvae. Syst. Entomol. 20, 337-370.

Seifert, B., 2009. Cryptic species in ants (Hymenoptera: Formicidae) revisited: we need a change in the alpha-taxonomic approach. Myrmecol. News 12, 149-166.

Solis, D.R., Fox, E.G.P., Rossi, M.L., Bueno, O.C., 2009. Description of the immatures of workers of the weaver ant, Camponotus textor (Hymenoptera: Formicidae). Sociobiology 54, 541-559.

Solis, D.R., Fox, E.G.P., Rossi, M.L., Moretti, T.C., Bueno, O.C., 2010. Description of the immatures of workers of the ant Camponotus vittatus (Hymenoptera: Formicidae). Florida Entomol. 93, 265-276.

Tamura, K., Peterson, D., Peterson, N., Stecher, G., Nei, M., Kumar, S., 2011. MEGA5: Molecular Evolutionary Genetics Analysis using maximum likelihood, evolutionary distance, and maximum parsimony methods. Mol. Biol. Evol. 28, 2731-2739.

Thompson, J.D., Higgins, D.G., Gibson, T.J., 1994. CLUSTAL W: Improving the sensitivity of progressive multiple sequence alignment through sequence weighting, positionspecific gap penalties and weight matrix choice. Nucleic Acids Res. 22, 4673-4680.

Vanden Dool, H., Kratz, P.D.J., 1963. A generalization of retention index system including linear temperature programmed gas-liquid partition chromatography. J. Chromatogr. $11,463-471$.

Vander Meer, R.K., Lofgren, C.S., 1988. Use of chemical characters in defining populations of fire ants, Solenopsis saevissima complex (Hymenoptera: Formicidae). Florida Entomol. 71 (3), 323-332.

Wheeler, G.C., Wheeler, J., 1976. Ant larvae: Review and Synthesis. Mem. Entomol. Soc. Washington 7, 1-108. 